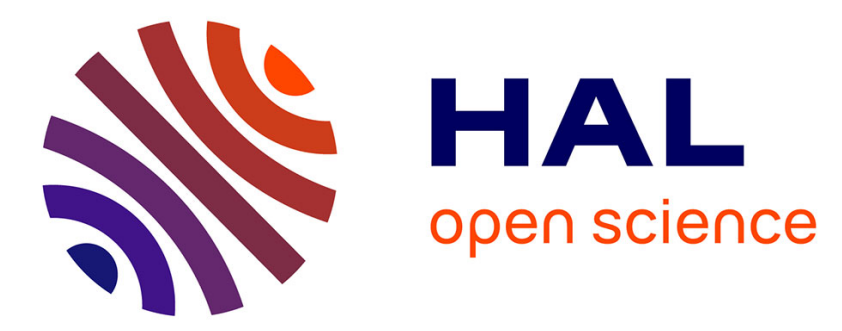

\title{
Les débuts de l'utilisation des inscriptions médiévales pour la recherche historique en France
}

\author{
Robert Favreau
}

\section{To cite this version:}

Robert Favreau. Les débuts de l'utilisation des inscriptions médiévales pour la recherche historique en France. Bibliothèque de l'École des chartes, 2015, 173, pp.465-486. halshs-03219568

\section{HAL Id: halshs-03219568 \\ https://shs.hal.science/halshs-03219568}

Submitted on 6 May 2021

HAL is a multi-disciplinary open access archive for the deposit and dissemination of scientific research documents, whether they are published or not. The documents may come from teaching and research institutions in France or abroad, or from public or private research centers.
L'archive ouverte pluridisciplinaire HAL, est destinée au dépôt et à la diffusion de documents scientifiques de niveau recherche, publiés ou non, émanant des établissements d'enseignement et de recherche français ou étrangers, des laboratoires publics ou privés. 
Les débuts de l'utilisation des inscriptions médiévales pour la recherche historique en France

On peut trouver de premières mentions d'inscriptions médiévales dans les travaux historiques du XVIe siècle, mais c'est vraiment à partir du XVIIe siècle que les inscriptions médiévales commencent à être des compléments réguliers dans les histoires de villes ou de provinces, les biographies et les généalogies, les travaux d'histoire de l'Église. C'est une tendance qui apparaît générale en Europe, si l'on pense, pour l'Espagne, au bénédictin Prudencio de Sandoval, qui publie en 1601 la Primera parte de las fundaciones de los monasterios del glorioso padro san Benito ${ }^{1}$, au bénédictin Antonio de Yepes ${ }^{2}$, dont l'Historia general de la orden de San Benito, en sept volumes, paraît à Irache en 1609 et 1610, puis à Valladolid en 1613, 1615, 1617, et, après sa mort 1621, ou au cistercien Angel Manrique, dont les quatre volumes d'Annales cistercienses sont publiés à Lyon de 1642 à 16593, tous ouvrages qui contiennent des dizaines d'inscriptions. Pour l'Italie s'impose le nom de l'oratorien Cesare Baronio, dit Baronius, qui fut cardinal et aurait pu être pape, et a laissé, dans les douze volumes de ses Annales ecclesiastici, parus de 1588 à 1607 à Rome et couvrant l'histoire de l'Église des origines à 1198, une œuvre capitale, où les inscriptions sont régulièrement utilisées 4 .

Les historiens de Toulouse fournissent un bon exemple de l'utilisation régulière des inscriptions parmi leurs sources. Antoine Noguier, "toulousain", publie en 1559 une Histoire tolosaine dans laquelle il rapporte une vingtaine d'inscriptions, par exemple les épitaphes des comtes dans l'enfeu des comtes de Toulouse à Saint-Sernin, ou l'inscription qui accompagne la statue de Saturnin à la cathédrale Saint-Etienne. Conseiller du roi au parlement de Toulouse Guillaume de Catel publie en 1623 une Histoire des comtes de Toulouse, où il cite lui aussi différentes inscriptions, rapportant même une épitaphe métrique en gascon du XIIIe siècle qu'il n'a pu réussir à trouver ; dans ses Mémoires de l'histoire du Languedoc, en 1633, ce sera près de 50 inscriptions dont Catel donnera le texte. "L'ample description de la ville de Toulose..." par Léon Godefroy en $1638^{5}$ ne manque pas de citer et localiser une série d'inscriptions, et maître Raymond Daydé, greffier audiencier de la seneschaussée et cour presidiale de Tolose, enrichit son Histoire de Saint-Sernin ou l'incomparable trésor de son église abbatiale de Tolose, en 1661, d'une vingtaine d'inscriptions, sans négliger des textes "de fort difficile lecture" ou comportant "quelques lettres tronquées". Le médecin Jean Bernier6 a recopié peu d'inscriptions dans son Histoire de Blois parue en 1682, mais il nous a transmis les planches gravées de deux inscriptions importantes aujourd'hui disparues ${ }^{7}$.

On comprend bien l'intérêt de recueillir les épitaphes pour les historiens qui se préoccupent de biographie. Ainsi Pierre Frizon né à Reims en 15868, d'abord jésuite, puis docteur en théologie, agrégé au collège de Navarre, vicaire général de la Grande Aumônerie de

\footnotetext{
1 - Notice de J. Goñi dans le Diccionario de historia eclesiastica de España, IV, Madrid, 1975, p. 2174-2179.

2 - Notice de J. Pérez de Urbel, ibid., IV, p. 2788-2790.

3 - Notice de P. Guérin, ibid., II, Madrid, 1972, p. 1407-1408 ; Vicente Garcia Lobo, "Les Annales de Angel Manrique y la cultura escrita. Planteamiento general...", Humanismo y císter, dir. G. Morocho Gayo, León, 1996, p. 391-404 ; Encarnación Martín López, "Les inscriptions médiévales dans les Annales de Manrique, contribution à la possibilité d'une épigraphie cistercienne", Cahiers de civilisation médiévale, 52, 2009, p. 395-413.

4 - Notice de A. Molien, dans le Dictionnaire d'histoire et de géographie ecclésiastique, t. 6, Paris, 1932, col. 871-882.

5 - Paris, Bibl. de l'Institut, Collection Godefroy ms. 220, fol. $7 \mathrm{v}^{\circ}-15 \mathrm{v}^{\circ}$.

6 - Notice de M. Prévost dans le Dictionnaire de biographie française, VI, 1954, c. 114.

7 - P. 292-293 et 301-302.

8 - Notice de H. Beylard, Dictionnaire de biographie française, t. 14, 1979, c. 1317.
} 
France, compile en 1629 une histoire des cardinaux français, revue et augmentée en 1638, la Gallia purpurata. Étienne Baluze en montrera les erreurs dans son Anti-Frizionius, mais on notera ici que Frizon insère dans son ouvrage plus d'une centaine d'épitaphes, à commencer par les papes d'origine "française", Sylvestre II, Léon IX, Clément IV, Martin V, Clément V, Innocent VI, Grégoire XI. Les frères jumeaux poitevins Scévole II et Louis de Sainte-Marthe, historiographes du roi, commencent la publication de la Gallia christiana, qu'achèveront les fils de Scévole, Pierre Abel et Nicolas. Les quatre volumes de 1656 consacrés aux archevêques (I), aux évêques (II et IV), et aux abbés (III) comportent un très grand nombre d'épitaphes, ce que continueront et amplifieront les rééditions des XVIIIe et XIX"e siècles ${ }^{9}$.

François Roger de Gaignières, né en 1642 en Nivernais, écuyer du duc de Guise puis au service de Mlle de Guise et logé jusqu'en 1710 à l'hôtel de Guise, n'est pas un épigraphiste, mais, comme il s'intéressait particulièrement à la généalogie des familles, grands officiers, évêques, abbés, il a fait dessiner un grand nombre de pierres tombales avec leurs inscriptions, et par là son apport à l'épigraphie est considérable. Dès les années 1680 il a constitué une collection notable d'épitaphes. À partir de 1695 il a parcouru l'Ouest de la France, de la Normandie au Poitou, la Bourgogne et la région parisienne, accompagné de son valet, Barthélemy Remy, et d'un artiste dessinateur, Louis Boudan, qu'il logeait dans sa maison et employait presque toute l'année. Outre les dessins de monuments ou de villes, Boudan, qui était devenu un bon "paléographe", a dessiné un grand nombre de pierres tombales gravées, en relevant avec soin les épitaphes. Gaignières a fait don de sa collection à la bibliothèque du roi en 1711 , et est mort en 1715. La collection Gaignières comprenait 7500 dessins et environ 2500 tombes. Malheureusement ce fut Pierre de Clairambault (1651-1740) qui fut chargé de la classer. Clairambault, d'abord conseiller à la marine, avait été pourvu en 1698 de la charge de généalogiste des ordres du roi. Il ôta des portefeuilles de Gaignières ce qui pouvait intéresser ses collections généalogiques. Il garda pour lui 1859 dessins, qui furent achetés par l'antiquaire anglais Richard Gough, lequel les céda à Douce, et ils passèrent alors à la bibliothèque bodléienne d'Oxford. En 1864 les dessins de Gaignières ont été retirés de la collection Clairambault et ont été remis au Cabinet des estampes ; vers la même date le gouvernement chargea M. Frappier de copier, pour le département des estampes, les dessins de Gaignières conservés à Oxford ${ }^{10}$. Les "tombeaux" de la collection Gaignières ont été heureusement publiés ${ }^{11}$, ce qui permet une parfaite appréhension du fonds, même si le format réduit des reproductions ne permet pas toujours la lecture des épitaphes.

Les recherches généalogiques ont conduit à des compilations d'inscriptions, principalement des épitaphes, sans forcément aboutir à une publication, et nos bibliothèques sont riches de manuscrits "épigraphiques" qui restent à explorer systématiquement. Le ms 613 de la collection Lorraine à la Bibliothèque nationale de France, attribué à Nicolas Bousmard, chanoine de l'église de Verdun, recueil de généalogies, chartes, épitaphes concernant l'histoire des familles de Lorraine, doit être une des plus anciennes puisque réalisée de 1573 à 1578 . Notable aussi est la collection Chifflet à la Bibliothèque de la ville de Besançon, XVIe-XVIIe siècles, aussi bien pour Besançon que pour Flandres et Artois. A la Bibliothèque de Reims, c'est le ms. 1941, recueil d'épitaphes de Charles Drouin Regnault, curé de Bézannes, XVII e siècle, à celle d'Arras le ms. 756, épitaphes et armoiries sur tombeaux (Lille, Douai, Orchies), du XVIe siècle ou le ms. 652, recueil d'épitaphes d'Est. Le Pez (Flandre, Picardie, Artois, XVII siècle). Et naturellement il faut revenir à la Bibliothèque nationale de France, ms. fr. 4901 pour la Basse-

\footnotetext{
9 - Paris, 1715-1865, 16 vol. in-folio.

10 - Léopold Delisle, Le cabinet des manuscrits, I, Paris, 1868, p. 335-356.

11 - Jean Adhémar et Gertrude Dordor, "Les tombeaux de la collection Gaignières, dessins d'archéologie du XVII" siècle", Gazette des Beaux-Arts, juillet-septembre 1974, p. 1-193 (n 1-1085), juillet-septembre 1976, p. 1-128 ( ${ }^{\circ}$ 1086-1337), juillet-août 1977 ( $\mathrm{n}^{\circ}$ 1838-2029) ; Jean-Bernard de Vaivre, "Dessins inédits de tombes médiévales de la collection Gaignières", ibid., octobre 1986, p. 97-122, et novembre 1986, p. 141$182,248 \mathrm{n}^{\circ}$.
} 
Normandie, ms. fr. 8538, épitaphes d'Arras, ms. fr. 32705-32707, par Jean Megret, pour Paris, ms. fr. 32944 pour Paris, tous manuscrits du XVII e siècle, et ce qui nous ramène à Pierre Clairambault, ms. fr. 8216-8240, recueil d'épitaphes formé par lui en partie avec les débris de la collection Gaignières, ou ms. 941-947, collection Clairambault pour une grande partie de la France du Nord.

L'histoire de l'Église fait explicitement partie des travaux auxquels sont conviés les moines bénédictins de la congrégation de Saint-Maur. Déjà les "divers tombeaux et épitaphe de l'église, cloître et chapitre" font partie de l'Histoire de l'abbaye royale de Saint-Ouen de Rouen par un religieux de la congrégation de Saint-Maur en 166212. Dans son histoire de l'Église de Reims, dom Guillaume Marlot retient plus d'une vingtaine d'épitaphes ${ }^{13}$. À la fin du XVII e siècle "l'utilisation systématique des sources non littéraires, médailles, inscriptions, lois, vient donner au travail historique, fondé jusqu'ici sur le recours aux textes narratifs, une visage nouveau"14, et de passage à Paris dans la première moitié du XVIIIe, Scipione, marquis de Maffei, nommé membre honoraire étranger de l'Académie des Inscriptions, propose d'installer dans une grande galerie du Louvre un musée lapidaire pour recueillir les inscriptions éparses à Paris et aux alentours ${ }^{15}$. La Méthode pour la recherche des manuscriptz invite à "ne rejeter rien, quand ce ne seroit qu'un épigramme d'un distique", et parmi les projets des maurisres pour l'illustration et la gloire de l'Église gallicane figure "un nécrologe général avec les sépulcres, les épitaphes et les inscriptions anciennes et modernes"16. Bref l'histoire se veut totale, et si l'intérêt est encore surtout porté sur l'Antiquité par les chercheurs humanistes, les Mauristes, attachés à l'histoire de l'Église, se penchent principalement sur l'histoire médiévale. C'est ainsi que dom Edmond Martène (1654-1739) qui avait grandement œuvré à recueillir des matériaux pour la rédaction de la nouvelle Gallia christiana, publie en 1717-1724, avec son compagnon d'érudition dom Ursin Durand, un Voyage littéraire de deux religieux bénédictins de la congrégation de Saint-Maur ; ils y annoncent d'entrée qu'en premier lieu on y trouvera "quantité de pièces, d'inscriptions et d'épitaphes servant à éclaircir l'histoire et les généalogies des anciennes familles".

Les bénédictins deviennent au XVIII ${ }^{\mathrm{e}}$ siècle, à l'invitation du pouvoir, les historiens officiels des provinces ${ }^{17}$. Ils y feront régulièrement place aux inscriptions, même s'ils ne leur réservent que fort peu de planches. C'est ainsi que dans les cinq volumes de l'Histoire générale de Languedoc (1730-1745), dom Vaissette donne 3 fac-similés des épitaphes des comtes de Toulouse à Saint-Sernin alors qu'il relève 80 inscriptions antiques et donne 8 planches de sceaux. Dom Urbain Plancher, dans ses quatre volumes de l'Histoire générale et particulière de la Bourgogne (1739-1781) se contente d'une planche pour quatre plates-tombes à inscriptions. Dom Augustin Calmet, qui n'est pas mauriste mais président de la congrégation de Saint-Vanne, cite dans son Histoire ecclésiastique et civile de la Lorraine ${ }^{18}$, bon nombre d'épitaphe des évêques de Toul et des ducs de Lorraine.

Les Mauristes ne se cantonnent pas aux histoires de province. Dom Bernard de Montfaucon (1655-1741), d'abord soldat, entre chez les Mauristes en 1675 : il devient professeur, connaisseur du grec, des langues orientales, familier des bibliothèques, érudit reconnu, reçu en 1719 par l'Académie des inscriptions. L'objet de ses Monumens de la monarchie

\footnotetext{
12 - P. 211-214 ; voir aussi p. 197. Ce moine s'appelle Pommeraye.

13 - Metropolis Remensis historia, I, Lille, 1666 ; II, Reims, 1679, posthume.

14 - Bruno Neveu, Érudition et religion aux XVII' et XVIII' siècles, Paris, 1994, p. 26.

15 - Ibid, p. 128-129.

16 - François Dolbeau, "Quelques instruments de travail chez les Mauristes", Comptes rendus de l'Académie des Inscriptions et Belles-Lettres, année 2007, p. 1743.

17 - Bruno Neveu, op. cit., p. 183.

18 - 4 volumes, Nancy, 1728.
} 
française ${ }^{19}$ est d'abord iconographique, mais il réserve une longue série de planches aux inscriptions de la tapisserie de Bayeux, aux tomes I et II, et fournit diverses épitaphes, avec planches. Un long chapitre, avec planches, est réservé par dom Tassin et dom Toustain aux écritures gravées, empreintes, tracées ou peintes sur les métaux, les marbres, les pierres, l'ivoire au tome II de leur Nouveau traité de diplomatique ${ }^{20}$, et l'on retrouve les inscriptions dans leur dernier volume. Dom Antoine Rivet de La Grange (1683-1749) a été l'initiateur de l'Histoire littéraire de la France, et fut le principal auteur des huit premiers volumes ; deux autres mauristes, dom Charles Clémencet et dom François Clément mèneront la publication jusqu'au $12^{\mathrm{e}}$ volume (1763) ; les épitaphes sont partie ordinaire des notices biographiques des différents auteurs recensés siècle après siècle.

Il n'y a pas eu que les Mauristes à s'intéresser à l'histoire et à y inclure des sources épigraphiques. Etienne Baluze, né à Tulle en 1630, mort à Paris en 1718, fut un simple clerc, mais pourvu de nombreux bénéfices. D'abord au service du savant archevêque de Toulouse, Pierre de Marca, puis de l'archevêque d'Auch, il devint en 1667 bibliothécaire de Colbert, et il sera en 1670 professeur de droit canon au collège royal (collège de France). Ses travaux sont solidement documentés. Ses manuscrits seront acquis par la bibliothèque du roi21. Dans son Histoire générale de la maison d'Auvergne justifiée par chartes, titres, histoires anciennes et autres preuves authentiques, il cite régulièrement les inscriptions, surtout des épitaphes, et en donne les planches $^{22}$. Jean-Aimar Piganiol de La Force, né à Aurillac en 1673, mort à Paris en 1753, a été pendant quarante ans précepteur des pages du comte de Toulouse. Parmi ses œuvres sa Description de la ville de Paris et de ses environs, parue en 1742, est particulièrement riche en relevés d'épitaphes. Conscient de la nouveauté de son propos, il s'en défendra : "Peut-être que des lecteurs frivoles me blameront d'avoir rapporté tant d'épitaphes, et ceux qui tirent parti de tout bord m'en sauront gré. Ils savent mieux que moi que les épitaphes servent infiniment à la chronologie en constatant les dates, et qu'elles répandent aussi beaucoup de jour sur l'Histoire"23.

Jean-François Dreux-Duradier (1714-1780), fut d'abord lieutenant particulier civil et criminel de Châteauneuf-en-Thimerais, sa ville natale. Il fut exilé par lettre de cachet à Poitiers en 1749, vendit sa charge en 1750 et se fixa à Paris avant de se retirer en 1760 à la campagne à Saint-Eliph (Eure-et-Loir). Grâce à un héritage conséquent il avait pu se consacrer aux études. Il a publié notamment en 1754, en cinq volumes, une Bibliothèque historique et critique du Poitou, où les inscriptions sont régulièrement citées. Les inscriptions étaient considérées à cette époque comme pouvant intéresser les personnes cultivées, curieuses d'Histoire. Onze articles des Affiches du Poitou, journal hebdomadaire publié de 1773 à 1789 par Jouyneau Desloges, ont pour sujets "épitaphes" ou "inscriptions", et un correspondant écrit : "Il seroit bien a désirer que l'on relevât avec soin et que l'on consignât dans un dépôt public toutes les inscriptions qui se trouvent dans les églises, les cimetières, les auditoires, les maisons particulières, les places et les édifices publics : le temps les use, les réparations les détruisent, et il ne reste que des fragments qui ne serviront qu'à embrouiller l'histoire et à exercer inutilement la sagacité des académiciens des siècles à venir"24.

Les fonds manuscrits de nos bibliothèques conservent de nombreux relevés d'inscriptions, et parfois des recueils conséquents. Le ms. 207 de la Société des Antiquaires de Picarde est consacré aux épitaphes et inscriptions des personnes illustres et autres qui se trouvent dans les

\footnotetext{
19 - Paris, 1729-1733.

20 - Paris, 1750-1765, 6 vol. ; chap. XI du vol. II, p. 535-695.

21 - Notice de A. Martin, Dictionnaire de biographie française, V, Paris, 1951, c. 23-25.

22 - Tome I, Paris, 1708, p. 5, 204 (pl.), 304 (pl.), 312 (pl.), 333 (pl.), 343 (pl.), 351 (pl.).

23 - Émile Raunier, Épitaphes du Vieux Paris, I, Paris, 1890, p. LIII.

24 - Régis Rech, "Histoire et presse à la fin de l'Ancien Régime : l'exemple des Affiches du Poitou", Revue historique du Centre-Ouest, VIII, 2009, p. 262-263.
} 
églises de Picardie. Oeuvre de Nicolas de Villiers il a été analysé dans la revue de cette société25. Jean-Raymond de Veras, chanoine de Saint-Pierre d'Avignon, a rassemblé en 1750 en un volume de 333 folios les épitaphes et inscriptions des églises d'Avignon ${ }^{26}$; en 1782 notre chanoine écrivait : "Il est à noter que ce recueil devient et deviendra de plus en plus appréciable, par la grande quantité d'inscriptions et d'épitaphes que l'on détruit tous les jours par les nouvelles décorations et autres changements que l'on fait continuellement aux églises". On lui doit aussi un recueil, complété par Esprit-Claude-François Calvet, pour le Comtat Venaissin ${ }^{27}$. L'abbé Tranchant a réuni en 1764 les inscriptions de Cambrai28, et messire Ferdinand-Ignace Maloteau, chevalier, seigneur de Villerode, conseiller au parlement de Flandre, chef des magistrats de Valenciennes, a recueilli les anciens tombeaux, épitaphes et sépultures de la plupart des églises du Pays-Bas, d'Artois, de Hainaut dans la première moitié du XVIIIe siècle, en trois recueils, au total 952 pages pour près de 400 localités $^{29}$. Les épitaphes et inscriptions d'Orléans et de son diocèse, relevées par Daniel Polluche, ont été mises en ordre en 1780 par dom Fabre, bibliothécaire de la Bonne Nouvelle ${ }^{30}$. La Bibliothèque du Sénat conserve trois volumes, au total 931 folios, sur les tombeaux et épitaphes des églises de Paris et de ses faubourgs ${ }^{31}$, et dom P.N. Pinchart, chanoine régulier, a réuni, en plusieurs recueils, de "très nombreuses épitaphes" et inscriptions commémoratives de Reims ${ }^{32}$.

Aubin-Louis Millin (1759-1818), fils d'un intendant aux vivres à l'armée, et d'une mère de famille noble de Bretagne, avait assez de fortune pour se consacrer à l'étude, notamment de la géologie et de la botanique, ses premières amours. Mais il se rallia avec enthousiasme à la Révolution, avant de condamner les excès de la Terreur, ce qui lui valut de se contenter d'un emploi subalterne dans les transports militaires, et un temps un emprisonnement à SaintLazare. Réduit à la pauvreté il va passer de chef de division au Comité de l'Instruction publique à l'enseignement de l'histoire et finalement à un poste de conservateur du Cabinet des antiques et médailles. C'est ainsi que peu à peu il abandonnera la botanique pour l'archéologie. Au cours de ses voyages en France et en Italie il a recueilli de nombreuses inscriptions (un millier pour l'Italie). Dans ses Antiquités nationales ${ }^{33}$ on peut trouver un très grand nombre d'inscriptions qui sans lui nous seraient totalement inconnues. Dès sa mort des notices nécrologiques élogieuses lui ont été consacrées dans les Mémoires de la Société royale des Antiquaires de France ${ }^{34}$ et les Mémoires de l'Académie des Inscriptions et Belles-Lettres ${ }^{35}$.

On s'était surtout intéressé aux XVIIe et XVIIIe siècles, notamment en France du Sud, aux inscriptions antiques. Au cours des années 1830-1840 on discute beaucoup, dans les cercles

\footnotetext{
25 - [Soyez], "L'épitaphier de Villers-Rousseville", Bulletin de la Société des Antiquaires de Picardie, t. XVIII, 1892-1894, p. 25-42.

26 - Bibliothèque d'Avignon, ms. 1738.

27 - Ibid., ms. 2376.

28 - Bib. de Cambrai, ms. 1049 (941).

29 - Bibl. de Douai, mss. 966-968.

30 - Bibl. d'Orléans, ms. 621 (464), 354 folios.

31 - ms. 949-951.

32 - Bibl. de Reims, ms. 1148-1150, 1153-1154.

33 - Antiquités nationales ou Recueil de monuments pour servir à l'histoire générale et particulière de l'empire français tels que tombeaux, inscriptions, statues, vitraux, fresques, etc., tirés des abbayes, monastères, châteaux et autres lieux devenus domaines nationaux, Paris, 1791, 5 vol. in$4^{\circ}$.

34 - En 1820, p. 52-69, par P.-R. Anguis.

35 - Notice plus récente de H. Leclercq, Dictionnaire d'archéologie chrétienne et de liturgie, XI-1, Paris, 1933, c. 1196-1199.
} 
parisiens, de la réalisation d'un Corpus inscriptionum latinarum ${ }^{36}$. Les discussions firent prévaloir le classemenet géographique sur le classement chronologique. Apparait alors le mot "épigraphie" pour désigner la science des inscriptions. Finalement ce sera l'Académie de Berlin qui pilotera le projet.

Mais au cours du XIXe siècle ce sont les amateurs passionnés, les "antiquaires", qui vont faire progresser la recherche épigraphique, non les professionnels de l'Histoire. Edmond Le Blant (1818-1897) en est un bon exemple. Après avoir fait des études de droit, obtenu le titre d'avocat, il était entré comme commis au Ministère des Finances, dans le service des douanes. Un voyage à Rome et la rencontre de Jean-Baptiste de Rossi en 1847 l'amenèrent à se consacrer à un recueil général des inscriptions chrétiennes de la Gaule. Le plus ancien marbre daté en Gaule était de 334, et deux autre seulement appartenaient au IVe siècle. Le travail de Le Blant va donc porter essentiellement sur les Ve-VII ${ }^{e}$ siècles. Son emploi lui laissait une grande liberté, sous l'autorité bienveillante du directeur général, son beau-frère. Il voyage donc par toute la Gaule, c'est-à-dire la France et les pays mosans-rhénans, visite musées, églises, cimetières, dépouille les recueils des XVIIe et XVIIIe siècles, recopie l'index manuscrit des inscriptions chrétiennes de Seguier, correspond avec les érudits locaux. En 1856 et 1865 il publie les deux volumes des Inscriptions chrétiennes de la Gaule antérieures au VIIIe siècle, et il leur donnera un supplément en 1892. La préface du tome II, revue et amplfiée, devient un Manuel d'épigraphie chrétienne d'après les marbres de la Gaule en 1869. De nombreux articles, une synthèse sur la "paléographie des inscriptions latines du IIIe siècle à la fin du VII ${ }^{\mathrm{e}}$ siècle" 37 complètent cet apport essentiel à l'épigraphie chrétienne qui valut au commis des douanes d'entrer à l'Académie des Inscriptions et Belles-Lettres.

Dans le même temps toute la société, dans le grand mouvement romantique, se passionnait pour le Moyen Âge. L'émotion de Châteaubriand, dans son Génie du christianisme (1802) devant la beauté des cathédrales gothiques, le succès de Notre-Dame de Paris (1831) de Victor Hugo, en étaient des déclencheurs, tout autant que les six volumes de l'Histoire de France (jusqu'à Louis XI) de Jules Michelet, publiés de 1833 à 1846. Tandis que les historiens découvraient l'énorme apport des fonds d'archives réunis à la Révolution dans des dépôts publics, et que les maîtres publiaient des histoires "nationales" ou des histoires de règne pour lesquelles l'épigraphie était de peu de secours, des sociétés savantes étaient créées partout en province et leurs adhérents recueillaient et publiaient les témoignages des inscriptions, des plus humbles aux plus savantes.

Arcisse de Caumont (1801-1873) s'était intéressé d'abord à la géologie. Sa fortune lui permettait de suivre ses goûts ; et il va bientôt se passionner pour l'archéologie et le Moyen Âge. Il est en 1823 un des fondateurs, à Caen, de la Société des Antiquaires de Normandie. En 1832 il organise une ligue des archéologues de Poitou, Maine, Touraine et Normandie pour défendre le baptistère Saint-Jean de Poitiers, que les édiles municipaux voulaient détruire afin de tracer une route droite entre le pont neuf et le centre ville. À cette association fortuite est substituée en 1834 la Société française d'archéologie avec le Bulletin monumental et les Congrès archéologiques. Caumont publie de 1830 à 1841 son Cours d'antiquités monumentales, où il écrit : "Les inscriptions tumulaires des XIe et XIIe siècles sont un curieux sujet d'étude que je ne saurais trop vous recommander : on a presque complètement négligé de relever ces inscriptions dont un grand nombre a malheureusement péri ; il est grand temps de recueillir celles qui nous restent". Et de fait Caumont mentionnera régulièrement dans ses travaux les inscriptions qu'il découvrait

\footnotetext{
36 - R. Favreau, "L'épigraphie médiévale : naissance et développement d'une discipline", Comptes rendus de l'Académie des Inscriptions et Belles-Lettres, année 1989, p. 329-330.

37 - Revue archéologique, t. 29-31, 1896-1897, et à part, Paris, 1898, 68 p.
} 
dans les monuments ${ }^{38}$. Et dans le Bulletin monumental on trouvera régulièrement découverte ou étude d'inscriptions médiévales.

Dans le Midi rien ne prédisposait Joseph Léonard, marquis de Castellane, né en 1761, à s'intéresser à l'épigraphie. Il suivit la carrière des armes et il était, en 1788, major en second du régiment Médoc-Infanterie. Il émigra en Allemagne, puis à Londres, où la peinture de camées lui permit de vivre honorablement. Revenu en France, il fut colonel de la garde nationale à Toulouse, maréchal de camp. En 1831 il fonda la Société archéologique du Midi de la France. On doit à ce septuagénaire trois études sur les "Inscriptions recueillies principalement dans le Midi de la France"39, soit un recueil de 316 inscriptions et 147 fac-similés qu'il a lithographiés de sa main. Il est mort en $1845^{40}$. La Société des Antiquaires de l'Ouest, fondée en 1834, a publié, en 1851, le Manuel d'épigraphie suivi du recueil des inscriptions du Limousin de l'abbé Texier, supérieur du petit séminaire du Dorat ${ }^{41}$. Si le Manuel correspond à une synthèse un peu prématurée, les 291 inscriptions recueillies, de l'époque romaine à 1848, même si elles n'épuisent pas le sujet, offrent une utile première recension. Il en sera de même avec l'Épigraphie du Haut-Poitou publié par la même société en 186442, 300 inscriptions de 140 à 1804, avec une série de planches. L'auteur, A. de Longuemar, militaire, puis "propriétaire", passionné de géologie, excellent dessinateur, s'est distingué comme un infatigable prospecteur du Moyen Âge en Poitou, où l'achat d'une propriété l'avait fixé.

Dès lors les publications de chercheurs locaux se font nombreuses. Louis de Bonnefoy (1816-1887), originaire de Perpignan, fixé à Toulouse à la fin de sa vie, qualifié de "propriétaire", publie de 1856 à 1868 dans le Bulletin de la Société agricole, scientifique et littéraire des Pyrénées-Orientales, une "Épigraphie roussillonnaise ou recueil des inscriptions des PyrénéesOrientales" de 332 numéros, fort bien documentée. Le prolixe monseigneur Xavier Barbier de Montault, infatigable chercheur -il a laissé plus de 300000 fiches-, publie en 1868-1869 alors qu'il est historiographe du diocèse d'Angers, une Épigraphie du département de Maine-et-Loire de près de 500 pages (969 inscription jusqu'au XVIIIe siècle) ; on trouvera de nombreux articles sur des inscriptions parmi les 4000 titres que compte sa bibliographie. Bibliothécaire de la ville de Saintes Louis Audiat a passé dix ans de sa vie à réunir la documentation d'une Épigraphie santone et aunisienne qui commence par les inscriptions gallo-romaines. La plus grande partie des inscriptions recueillies était inédite. Déjà l'auteur constatait qu'un certain nombre de textes qu'il avait vus avaient disparu. Le premier volume de son recueil est paru en 1870, mais l'incendie qui détruisit en 1871 la bibliothèque de Saintes nous prive du second volume ${ }^{43}$. À Alfred de Terrebasse on doit deux volumes des inscriptions de Vienne en $1875^{44}$. À ces recueils proprement épigraphiques il faudrait joindre les Statistiques monumentales qui se publient à cette époque, car elles enregistrent régulièrement les inscriptions, par exemple la Statistique monumentale du département du Cher, en 8 volumes, publiée à partir de 1875 par AlphonseLouis Buot de Kersers (1802-1880), un polytechnicien qui fit carrière comme ingénieur des ponts et chaussées dans ce département. Gabriel Dumay (1841-1913), docteur en droit, juge d'instruction à Autun, fut mis à la retraite d'office en 1883 et se fixa à Dijon où il s'occupa d'histoire ; on lui doit une Épigraphie bourguignonne, église et abbaye de Saint-Bénigne de Dijon,

\footnotetext{
38 - E. de Robillard de Beaurepaire, Monsieur de Caumont. Sa vie et ses œuvres, Rouen, 1998 ; M. Prévost, notice dans le Dictionnaire de biographie française, Paris, VII, 1956, c.1459-1460 ; G. de Cougny, "Monsieur de Caumont", Bulletin monumental, XXXIX, 1873, p. 327 et sq.

39 - Mémoires de la Société archéologique du Midi de la France, 2, 1834-1835 ; 3, 1836-1837 ; 4, 1840-1841.

40 - Auguste d'Aldéguier, "Eloge de M. le marquis de Castellane...", Mémoires de la Société archéologique du Midi de la France, V, De 1841 à 1847, p. 297-316

41 - Mémoires de la Société des Antiquaires de l'Ouest, année 1850.

42 - Mém. Soc. Antiq. Ouest, t. XXVIII, année 1863.

43 - Bulletin de la Société des Archives historiques de la Saintonge et de l'Aunis, IV, 1883-1884, p. 109.

44 - Inscriptions antiques et du Moyen Âge de Vienne en Dauphiné ; le second volume consacré aux Inscriptions du Moyen Âge antérieures au XVII siècle.
} 
parue en part à Paris et Dijon en $1882^{45}$. Avec Harold de Fontenay, on trouve un chartiste, qui fut conservateur du Musée d'Autun et longtemps bibliothécaire de la Société éduenne ; ses deux volumes d'Épigraphie autunoise parus en 1883 et 1886, ont d'abord été publiés, comme pour Dijon, dans la revue de la Société savante locale ${ }^{46}$. La commission départementale des monuments historiques du Pas-de-Calais, a eu le mérite de mener à bien la publication en 8 volumes d'une Épigraphie du département du Pas-de-Calais, en 1883-1892. Paul Quesvers, "homme de lettres", et Henri Stein, historien, publient de 1887 à 1903 en 4 volumes les Inscriptions de l'ancien diocèse de Sens d'après les estampages d'Edmond Michel. Le baron de Rivières, né en 1835, a fait des études de droit à Toulouse et étudié le dessin pour pouvoir dessiner les monuments. Il a, en fait, passé sa vie à étudier monuments et histoire locale, membre de la Société française d'archéologie et participant assidu des Congrès archéologiques, membre de la Société archéologique du Midi de la France. Il est l'auteur d'une "Épigraphie albigeoise ou recueil des inscriptions de l'arrondissement d'Albi" parue dans la Revue historique, scientifique et littéraire du département du Tarn en 1894 et 1895 et est mort au début du XXe siècle en son château de Rivières près de Gaillac ${ }^{47}$. On peut encore citer les Inscriptions anciennes de l'arrondissement de Vouziers ou relatives à la région, publiées à Reims en 1892 par le docteur H. Vincent, ou les Inscriptions de Notre-Dame de Reims, textes relevés sur les monuments originaux, de Charles-Henri Jadart (1847-1921), magistrat à Rethel puis à Reims, et bibliothécaire de la ville de Reims, publiées à Reims en 1907. On mentionnera pour terminer l'abbé Alphonse Angot (1844-1917), qui prit tôt le goût de l'histoire, et fut autorisé en 1885 à quitter le ministère paroissial pour se livrer à ses études. Ce sera notamment l'Épigraphie de la Mayenne, 2 volumes très documentés publiés en 1907 et recensant 1622 textes. "C'est surtout parce qu'elle est un auxiliaire de son histoire que j'ai entrepris l'Épigraphie d'un pays que j'aime". L'abbé Angot est un bon exemple de ces érudits cultivés amoureux de leur terroir.

À Paris aussi l'épigraphie médiévale faisait des progrès. L'abbé Jacque-Paul Migne (18001875) réservait dans les tables de sa Patrologie latine un index épigraphicus ${ }^{48}$. Le ministre de l'Instruction publique, Guizot, avait institué en 1834 un Comité "chargé de concourir à la direction et à la surveillance des recherches et publications " sur les documents inédits relatifs à l'histoire de France. Après des intitulés divers le comité prendra en 1858 le nom de Comité des travaux historiques et scientifiques. Le premier secrétaire de ce comité, nommé par Guizot, fut Alphonse-Napoléon Didron qui, après des études grecques et latines, enseignait l'histoire pour vivre, et que la lecture de Notre-Dame de Paris avait converti à l'archéologie et au Moyen Âge. Didron publia, à partir des envois des sociétés savantes, les quatre premiers volumes du Bulletin archéologique du Comité. Il fonda en 1844 Les Annales archéologiques, et eut le mérite de trouver dans les travaux encyclopédiques de Vincent de Beauvais une clé d'explication de l'iconographie. Dès le premier volume des Annales Auguste Moutié publiait un article intitulé "Epigraphie. Inscriptions du Moyen Âge et de la Renaissance", où il demandait à chaque antiquaire de se mobiliser pour dresser un "corps complet des inscriptions, qui seraient classées par siècles et par matières" ; il déplorait d'innombrables destructions, citant en exemple deux dalles seulement subsistant sur 200 en la cathédrale d'Amiens. Didron lui-même avait compté, en 1844, 526 pierres tumulaires, dont 251 entières, à Notre-Dame de Châlons-en-Champagne : depuis, écrivait-il en 1859, "on a pris les plus belles dalles pour faire des seuils de portes, et des marches d'escaliers, les autres on les a découpées en pavés pour faire des trottoirs... ou débitées en moellons pour bâtir les mauvaises baraques de notre temps". Le ministre Salvandy avait voulu nommer Didron sous-bibliothécaire au département des manuscrits de la Bibliothèque royale, mais la nomination ne se fit pas en raison de "l'omnipotence des conservateurs". Les

\footnotetext{
45 - Extr. des Mémoires de la Commission des antiquités du département de la Côte-d'Or, 10, 1878-1884.

46 - Mémoires de la société éduenne, nouv. série, t. 7 à 16, 1878-1888.

47 - Notices nécrologiques dans cette même revue, XXVI, 1909, p. 175-177, et dans le Bulletin Monumental, 72, 1908, p. 545-547.

48 - Patrologie latine, t. 221, 1864, index CCXXVIII, col. 607-624.
} 
Annales archéologiques ont régulièrement fait place aux inscriptions, et leur succès est étroitement associé au nom de Didron ${ }^{49}$.

Ferdinand de Guilhermy (1809-1878) avait été initié au Moyen Âge et à ses monuments par son père. Après des études de droit il entra au ministère des finances (1829) et fut nommé en 1847 conseiller référendaire à la Cour des comptes. Il rédigea pour le Comité des arts et monuments quelque trois cents rapports sur l'établissement d'un inventaire général des richesses monumentales de la France. En 1848 il était chargé par le Comité des travaux historiques de rien moins qu'un recueil des inscriptions médiévales, et en 1856 une circulaire annonçait la publication d'un Recueil des inscriptions des Gaules et de la France. Dans sa jeunesse Guilhermy avait parcouru à pied les 450 villes, bourgs et villages de l'ancien diocèse de Paris, en prenant copie des inscriptions. Un bon dessinateur, Charles Fichet, rapporta de deux campagnes, en 1856 et 1862, 2000 estampes d'inscriptions. De 1873 à 1883 parurent, en cinq volumes, les Inscriptions de la France du Ve au XVIIe siècle. Ancien diocèse de Paris, dans la collection des Documents inédits sur l'histoire de France du Comité des travaux historiques et scientifiques, $3^{e}$ série, Archéologie ; après la mort de Lasteyrie, le travail avait été achevé par Robert de Lasteyrie. Guilhermy avait légué à la Bibliothèque nationale les 41 volumes de ses notes ${ }^{50}$.

En 1874 l'Académie des Inscriptions et Belles-Lettres avait ouvert un concours sur l'épigraphie. Robert de Lasteyrie de Saillant (1849-1921), sorti l'année précédente de l'École nationale des chartes, obtint le prix pour un recueil des inscriptions de la France comprises entre l'avènement de Pépin le Bref et la mort de Philippe Ier, plus de 900 inscriptions réunies en 18 cahiers, recueil qui n'a pas été conservé par l'Académie. En 1880 il devint professeur à l'École des chartes, où il enseignera l'archéologie jusqu'en 1910. Il déplorera dans les Études sur la sculpture française au Moyen Âge (1902) que "l'épigraphie du Moyen Âge ne soit guère en honneur parmi nos archéologues", alors, expliquait-il, que par exemple les inscriptions du cloître de Saint-Trophime d'Arles "permettent de déterminer avec une approximation très suffisante l'âge de ce cloître". Ce sera là longtemps l'attitude des archéologues français que de voir en l'épigraphie essentiellement une aide pour préciser la chronologie. Mais pour la première fois les inscriptions faisaient partie d'un enseignement au niveau des études supérieures.

La fondation à Poitiers en 1969 d'une équipe du C.N.R.S. chargée d'établir un recueil général des inscriptions médiévales de la France a apporté un changement important dans la situation de l'épigraphie médiévale. Il est clair que les travaux poursuivis depuis le XVIIe siècle par des érudits, des amateurs d'histoire locale, constituent un apport de grande importance. Il reste à poursuivre l'indispensable travail de publication des sources épigraphiques, et à faire prendre conscience aux enseignants et aux chercheurs du très large champ dans lequel les inscriptions peuvent apporter des compléments utiles à leurs recherches.

Robert Favreau

\footnotetext{
49 - Notice de F. de Guilhermy sur Didron dans les Annales archéologiques, XXV, 1865, p. 377-395.

50 - Bibliothèque nationale de France, n. acq. fr. 6094-6134; inventaire de ces volumes dans le Bulletin monumental, LXIX, 1905, p. 114-154. "Nécrologie du baron Ferdinand de Guilhermy" par J. de Laurière, dans le Bulletin monumental, t. 44, 1878, p. 406-408.
} 